\title{
Derivation of the Recursion Relation for the Feynman Diagrams of the CJT effective action
}

Chungku Kim

(Dated: November 13, 2018)

\begin{abstract}
We derive a new recursion relation to obtain the Feynman diagrams of the Cornwall-JackiwToumboulis(CJT) effective action by using the functional derivative identities. By using this recursion relation we show the two-particle-irreducibility of the Feynman diagrams of the CJT effective action by induction. We apply the recursion relation to obtain the Feynman diagrams of the CJT effective action up to the four-loop order in case of the bosonic field theory.
\end{abstract}

PACS numbers: 11.15.Bt, 12.38.Bx

\section{INTRODUCTION}

The effective action plays an important role in studies of the vacuum instability, the dynamical symmetry breaking and the dynamics of composite particles[1] for the given particle physics model. Among the several schemes for the nonperturbative evaluation of the effective action, the CJT effective action[2] was widely used as a resummation scheme which gives a self-consistent loop expansion of the effective action while preserving the symmetry of the original theory. In case of the connected and the one-particle-irreducible (1PI) effective action, the recursive generation of the Feynman diagrams was obtained for the multicomponent $\phi^{4}$-theory, QED and the scalar QED theories [3][4] [5][6][7][8][9] by using the functional integral identities $\int D \Phi \frac{\delta}{\delta \Phi} F[\Phi]=0$. In case of the CJT effective action which is the second Legendre transformation of the generating functional, the recursion relation was obtained by extracting the two-particle-irreducible (2PI) Feynman diagrams from the 1PI effective action [10]. Since the Feynman diagrams of the effective action obtained from the n-th Legendre transformation of the generating functional are not always the n-particle-irreducible (nPI) Feynman diagram [11], obtaining the recursion relation by extracting nPI Feynman diagrams is not possible when $n>2$.

Recently, we have derived a new method to obtain the recursion relation for the effective action by using the functional derivative identity [12]. This method can be extended to the case of the effective action obtained from the n-th Legendre transformation of the generating functional and in this paper, we apply this method to the CJT effective action. In Sec.II, we derive the recursion relation for the CJT effective action. We prove the two-particle-irreducibility of the CJT effective action by using the recursion relation. Then we apply to the case of Feynman diagrams up to the four-loop order effective action for the bosonic field theory. In Sec.III, we give some discussions and conclusions.

\section{A RECURSION RELATION FOR THE FEYNMAN DIAGRAMS OF THE CJT EFFECTIVE ACTION}

In this section, we will first derive a recursion relation for the Feynman diagrams of the CJT effective action for the bosonic field theory with the classical action

$$
S[\Phi]=\int\left\{\frac{1}{2} \Phi_{A} \Delta_{A B}^{-1} \Phi_{B}+S^{i n t}[\Phi]\right\} .
$$

where $S^{\text {int }}[\Phi]$ contains the higher vertices which appear in lattice regularization[13] as well as the cubic and the quartic interactions. In this paper, we use a notation where the capital letters contain both the space-time variables and the internal indices and the repeated capital letters mean both the integration over continuous variables and the sum over internal indices. For example, if the capital letter $A$ contains a space-time variable $x$ and the internal index $i$,

$$
J_{A} \Phi_{A} \equiv \sum_{i} \int d^{4} x J_{i}(x) \Phi_{i}(x) .
$$

The generating functional $W[J, K]$ is given by

$$
W[J, K]=-\hbar \ln \int D \Phi \operatorname{Exp}\left[-\frac{1}{\hbar}\left(S[\Phi]+J_{A} \Phi_{A}+\frac{1}{2 !} K_{A B} \Phi_{A} \Phi_{B}\right)\right]
$$


where the external source $K_{A B}$ is symmetric under the exchange of the indices. The functional derivative of the $W[J, K]$ with respect to the external sources are given by the classical field $\phi$ and the full propagator $\mathrm{G}$ as

$$
\begin{aligned}
\frac{\delta W[J, K]}{\delta J_{A}} & =\left\langle\Phi_{A}\right\rangle=\phi_{A}, \\
2 \frac{\delta W[J, K]}{\delta K_{A B}} & =\left\langle\Phi_{A} \Phi_{B}\right\rangle=\phi_{A} \phi_{B}+\hbar G_{A B},
\end{aligned}
$$

From (3) and (5) we can see that

$$
G_{A B} \equiv-\frac{\delta^{2} W[J, K]}{\delta J_{A} \delta J_{B}}
$$

By inverting (4) and (5), one can obtain the functionals $J[\phi, G]$ and $K[\phi, G]$. Then the CJT effective action is given by the second Legendre transformation of the generating functional as

$$
\Gamma[\phi, G]=W[J, K]-J_{A} \phi_{A}-\frac{1}{2} K_{A B}\left(\phi_{A} \phi_{B}+\hbar G_{A B}\right) .
$$

From (4),(5)and (7), one can obtain the following relations :

$$
\begin{aligned}
& \frac{\delta \Gamma[\phi, G]}{\delta \phi_{A}}=-J_{A}-K_{A B} \phi_{B}, \\
& \frac{\delta \Gamma[\phi, G]}{\delta G_{A B}}=-\frac{\hbar}{2} K_{A B} .
\end{aligned}
$$

and from (3) and (7), we obtain

$$
\exp \left\{-\frac{1}{\hbar} \Gamma[\phi, G]\right\}=\int D \Phi \exp \left\{-\frac{1}{\hbar}\left(S(\Phi)+J_{A}\left(\Phi_{A}-\phi_{A}\right)+\frac{1}{2} K_{A}\left(\Phi_{A} \Phi_{B}-\phi_{A} \phi_{B}-\hbar G_{A B}\right)\right)\right\} .
$$

By expanding the effective action $\Gamma[\phi, G]$ around $\hbar$, we obtain

$$
\Gamma[\phi, G]=\sum_{l=0} \hbar^{l} \Gamma^{(l)}[\phi, G]
$$

we can obtain the loop-wise expansion of $\Gamma[\phi, G][14]$. The first two terms are given by[2]

$$
\Gamma^{(0)}[\phi, G]=S[\phi], \Gamma^{(1)}[\phi, G]=\frac{1}{2} \operatorname{Tr} \ln G^{-1}-\frac{1}{2} \operatorname{Tr} G\left(G^{-1}-D^{-1}\right) .
$$

where

$$
D_{A B}^{-1}[\phi] \equiv \frac{\delta^{2} S[\phi]}{\delta \phi_{A} \delta \phi_{B}}
$$

Now consider the functional identities satisfied by the two sources $J[\phi, G]$ and $K[\phi, G]$

$$
\frac{\delta J_{A}}{\delta \phi_{C}} \frac{\delta \phi_{C}}{\delta J_{B}}+\frac{\delta J_{A}}{\delta G_{C D}} \frac{\delta G_{C D}}{\delta J_{B}}=\delta_{A B}
$$

and

$$
\frac{\delta K_{A E}}{\delta \phi_{C}} \frac{\delta \phi_{C}}{\delta J_{B}}+\frac{\delta K_{A E}}{\delta G_{C D}} \frac{\delta G_{C D}}{\delta J_{B}}=0
$$


By eliminating the term $\frac{\delta G_{C D}}{\delta J_{B}}$ from (14) and (15), we obtain

$$
\left(\frac{\delta J_{A}}{\delta \phi_{C}}-\frac{\delta J_{A}}{\delta G_{D E}} \Delta_{D E, P Q}^{-1} \frac{\delta K_{P Q}}{\delta \phi_{C}}\right) \frac{\delta \phi_{C}}{\delta J_{B}}=\delta_{A B}
$$

where

$$
\Delta_{A B, C D} \equiv \frac{\delta K_{A B}}{\delta G_{C D}}=-\frac{2}{\hbar} \frac{\delta^{2} \Gamma}{\delta G_{A B} \delta G_{C D}}
$$

We have used (9) in deriving the last line of the above equation. From (4) and (6), we can obtain

$$
\frac{\delta \phi_{C}}{\delta J_{B}}=\frac{\delta^{2} W[J]}{\delta J_{C} \delta J_{B}}=-G_{C B}
$$

From (8) and (9), we can obtain

$$
J_{A}=-\frac{\delta \Gamma[\phi, G]}{\delta \phi_{A}}+\frac{2}{\hbar} \frac{\delta \Gamma[\phi, G]}{\delta G_{A B}} \phi_{B}
$$

By substituting (9),(18) and (19) into (16), we can obtain

$$
-\frac{\delta^{2} \Gamma}{\delta \phi_{A} \delta \phi_{B}}+\frac{2}{\hbar} \frac{\delta \Gamma}{\delta G_{A C}}-2 \hbar \gamma_{A, D E} \Delta_{D E, P Q}^{-1} \gamma_{B, P Q}=-G_{A B}^{-1}
$$

where

$$
\gamma_{A, B C} \equiv \frac{1}{\hbar} \frac{\delta^{2} \Gamma}{\delta \phi_{A} \delta G_{D E}}
$$

Since $\Gamma^{(0)}$ does not depend on $G, \Delta$ and $\gamma$ can be expanded around $\hbar$ as

$$
\Delta_{A B, C D}=\sum_{l=0} \hbar^{l} \Delta_{A B, C D}^{(l)} \text { and } \gamma_{A, B C}=\sum_{l=0} \hbar^{l} \gamma_{A, B C}^{(l)}
$$

where

$$
\Delta_{A B, C D}^{(l)}=-2 \frac{\delta^{2} \Gamma^{(l+1)}}{\delta G_{A B} \delta G_{C D}} \text { and } \gamma_{A, B C}^{(l)}=\frac{\delta^{2} \Gamma^{(l+1)}}{\delta \phi_{A} \delta G_{D E}}
$$

From (12), we can obtain

$$
\Delta_{A B, C D}^{(0)}=-G_{A C}^{-1} G_{B D}^{-1}, \Delta_{A B, C D}^{-1(0)}=-G_{A C} G_{B D} \text { and } \gamma_{A, B C}^{(0)}=\frac{1}{2} S_{A B C}[\phi]
$$

where

$$
S_{A_{1} \ldots A_{N}}[\phi] \equiv \frac{\delta^{N} S[\phi]}{\delta \phi_{A_{1}} \cdots \delta \phi_{A_{N}}}
$$

Now, by using (12), we can check that order $\hbar^{0}$ term of (20) is satisfied and the higher order terms are given by

$$
G_{A B} \frac{\delta \Gamma^{(l)}[\phi, G]}{\delta G_{A B}}=\frac{1}{2} G_{A B} \frac{\delta^{2} \Gamma^{(l-1)}[\phi, G]}{\delta \phi_{A} \delta \phi_{B}}+\sum_{\substack{p, q, r \\ p+q+r=l-2}} G_{A B} \gamma_{A, D E}^{(p)} \Delta_{D E, P Q}^{-1(q)} \gamma_{B, P Q}^{(r)}(l \geq 2)
$$

Note that the result of the operation $G_{A B} \frac{\delta \Gamma^{(l)}}{\delta G_{A B}}$ is equal to multiplying each diagrams in $\Gamma^{(l)}$ by the number of its propagators $G$. Eq.(26) is the central result of this paper.

Before applying (26) to specific examples, let us show the two-particle-irreducibility of the Feynman diagrams of $\Gamma^{(l)}[\phi, G]$ by induction. For this purpose, let us assume that all the Feynman diagrams of the $\Gamma^{(k)}[\phi, G](k<l)$ are two-particle-irreducible. Since the operation $\frac{\delta}{\delta \phi}$ which appears at the first term of right hand side (RHS) of (26) 
changes only the vertex factors of the $\Gamma^{(l-1)}[\phi, G]$ which have 2PI Feynman diagrams by assumption ( see (32) and the second column of the Table 1 ), the first term of right hand side (RHS) of (26) consists of 2PI Feynman diagrams. The graphical representation of the second term of the RHS of (26) is given in Fig.1 and let us note the following properties of the $\gamma^{(k)}$ and $\Delta^{-1(k)}(k \leq l-2)$ which contribute to $\Gamma^{(l)}[\phi, G]$.

(A) $\gamma_{A, B C}^{(k)}(k \leq l-2)$ has a one-particle-irreducibility so that it can not be divided by two separate parts by cutting any one of the lines (see (34) and the third column of Table 1). This follows from (23), where the operation $\frac{\delta}{\delta \phi}$ changes the vertex factors and the operation $\frac{\delta}{\delta G}$ removes only one line from $\Gamma^{(k+1)}$ which have the two-particle-irreducible Feynman diagrams by assumption.

(B) $\Delta_{A B, C D}^{-1(k)}(k \leq l-2)$ does not have a diagram of the type given in Fig.2 so that it can not be divided by two separate parts containing the index $\mathrm{AB}$ and $\mathrm{CD}$ respectively. In order to see this, first note that $\Delta_{A B, C D}^{(k)}(k \leq l-2)$ does not have a diagram of the type given in Fig.2. This follows from (23) such that if we connect the points $A$ and $B$ of $\Delta_{A B, C D}^{(k)}$ with the propagator $G_{A B}$ and connect the points $C$ and $D$ with the propagator $G_{C D}$ , then we should obtain the 2PI diagram. If $\Delta_{A B, C D}^{(k)}$ have a diagram of the type given in Fig.2, this is impossible and hence $\Delta_{A B, C D}^{(k)}$ does not have a diagram shown in Fig.2 (see (35) and the fourth column of Table 1 ). Now, in order to see that $\Delta_{A B, C D}^{-1(k)}$ does not have a diagram of the type given in Fig.2 by induction, let us assume that $\Delta_{R S, C D}^{-1(m)}(m \leq k-1)$ does not have a diagram of the type given in Fig.2. By using (24), the perturbative expansion of the $\Delta_{A B, C D}^{-1(k)}$ is given by

$$
\Delta_{A B, C D}^{-1(k)}=-G_{A P} G_{B Q}\left(\Delta_{P Q, R S}^{(1)} \Delta_{R S, C D}^{-1(k-1)}+\Delta_{P Q, R S}^{(2)} \Delta_{R S, C D}^{-1(k-2)}+\ldots+\Delta_{P Q, R S}^{(k)} \Delta_{R S, C D}^{-1(0)}\right) .
$$

Since both the $\Delta_{A B, C D}^{(m)}(m \leq k)$ and $\Delta_{A B, C D}^{-1(k)}(m \leq k-1)$ of RHS of the above equation do not have a diagram of the type given in Fig.2, $\Delta_{A B, C D}^{-1(l)}$ does not have a diagram of the type given in Fig.2 also (see $(36)$ ).

From the properties (A) and (B) given above, it is clear that one can not divide the second term of RHS of (26) given in Fig.1 by two separated parts by cutting any two lines of the propagators. This completes the proof that all the Feynman diagrams of $\Gamma^{(l)}[\phi, G]$ are two-particle-irreducible.

Now, consider the $l=2$ case of $(26)$. In this case, the first term of (26) becomes

$$
\frac{1}{2} G_{A B} \frac{\delta^{2} \Gamma^{(1)}[\phi, G]}{\delta \phi_{A} \delta \phi_{B}}=\frac{1}{4} S_{A B C D}[\phi] G_{A B} G_{C D}
$$

and the second term of (26) becomes

$$
G_{A B} \gamma_{A, D E}^{(0)} \Delta_{D E, P Q}^{-1(0)} \gamma_{B, P Q}^{(0)}=-\frac{1}{4} S_{A D E}[\phi] S_{B P Q}[\phi] G_{A B} G_{D P} G_{E Q}
$$

In this paper we use the graphical representation where a line represents the propagator $G$ and a $n$-point vertex have the factor $S_{A_{1} \ldots A_{n}}$. Also a box with an capital letter represents the vertex which have indices that is not contracted with the propagators attached to it so that

$$
\overbrace{P}^{A . . B}=S_{A . . B P^{\prime} Q^{\prime} . . R^{\prime}} G_{P P^{\prime}} G_{Q Q^{\prime}} . . G_{R R^{\prime}}
$$

By substituting (28) and (29) into (26), we can obtain

$$
\Gamma^{(2)}[\phi, G]=-\frac{1}{12} S_{A Q R}[\phi] S_{B S P}[\phi] G_{A B} G_{P Q} G_{R S}+\frac{1}{8} S_{A B P Q}[\phi] G_{A B} G_{P Q}=-\frac{1}{12} \bigcirc+\frac{1}{8} \bigcirc \bigcirc .
$$

In order to consider (26) in case of $l=3$, we first note that

$$
\begin{aligned}
& \frac{\delta^{2} \Gamma^{(2)}[\phi, G]}{\delta \phi_{A} \delta \phi_{B}}=\frac{\delta^{2}}{\delta \phi_{A} \delta \phi_{B}}\left[-\frac{1}{12} \bigcirc+\frac{1}{8} \bigcirc \bigcirc\right]=\frac{\delta}{\delta \phi_{B}}\left[-\frac{1}{6} A \emptyset+\frac{1}{8} \bigcirc \text { 只 }\right] \\
& =-\frac{1}{6} A B @-\frac{1}{6} A \longmapsto B+\frac{1}{8} \bigcirc \text { 争. }
\end{aligned}
$$


and

From (23), we also obtain

$$
\frac{\delta \Gamma^{(2)}[\phi, G]}{\delta G_{A B}}=-\frac{1}{4} A \text { ф } B+\frac{1}{4} \bigodot_{A B}
$$

$$
\gamma_{A, B C}^{(1)}=\frac{\delta^{2} \Gamma^{(2)}[\phi, G]}{\delta \phi_{A} \delta G_{B C}}=-\frac{1}{4} A B \curvearrowleft g C-\frac{1}{4} B \curvearrowleft \varnothing A C+\frac{1}{4} \bigcap_{A B C}
$$

and

$$
\Delta_{A B, C D}^{(1)}=-2 \frac{\delta^{2} \Gamma^{(2)}[\phi, G]}{\delta G_{A B} \delta G_{C D}}=\frac{1}{2} A C \square-\square B D+\frac{1}{2} A D \square \square B C-\frac{1}{2} \quad \underset{\square}{A B C D}
$$

From (35), we can obtain the order $\hbar$ term of the inverse of the $\Delta$ as

$$
\begin{aligned}
& \Delta_{A B, C D}^{-1(1)}=-\Delta_{A B, P Q}^{-1(0)} \Delta_{P Q, R S}^{(1)} \Delta_{R S, C D}^{-1(0)}=-\frac{1}{2}{ }_{D}^{A} n_{C}^{B}-\left.\frac{1}{2}{ }_{C}^{A}\right|_{D} ^{B}+\left.\frac{1}{2} A\right|_{D} ^{B} C \\
& \text { three-loop effective action } \Gamma^{(3)},(26) \text { becomes }
\end{aligned}
$$

In case of the three-loop effective action $\Gamma^{(3)},(26)$ becomes

$$
\frac{\delta \Gamma^{(3)}[\phi, G]}{\delta G_{A B}} G_{A B}=\frac{1}{2} G_{A B} \frac{\delta^{2} \Gamma^{(2)}}{\delta \phi_{A} \delta \phi_{B}}+G_{A B} \gamma_{A, D E}^{(0)} \Delta_{D E, P Q}^{-1(1)} \gamma_{B, P Q}^{(0)}+2 G_{A B} \gamma_{A, D E}^{(1)} \Delta_{D E, P Q}^{-1(0)} \gamma_{B, P Q}^{(0)}
$$

By substituting (24),(32),(34) and (36) into (37), we obtain

$$
\Gamma^{(3)}[\phi, G]=-\frac{1}{48} \bigotimes+\frac{1}{8} \bigotimes-\frac{1}{24} \bigcirc-\frac{1}{12} \bigcirc+\frac{1}{48}
$$

Finally, in case of the four-loop effective action $\Gamma^{(4)},(26)$ becomes

$$
\begin{aligned}
\frac{\delta \Gamma^{(4)}[\phi, G]}{\delta G_{A B}} G_{A B}= & {\left[\frac{1}{2} \frac{\delta^{2} \Gamma^{(3)}}{\delta \phi_{A} \delta \phi_{B}}+2 \gamma_{A, P Q}^{(2)} \Delta_{P Q, R S}^{-1(0)} \gamma_{B, R S}^{(0)}+\gamma_{A, P Q}^{(1)} \Delta_{P Q, R S}^{-1(0)} \gamma_{B, R S}^{(1)}\right.} \\
& \left.+2 \gamma_{A, P Q}^{(1)} \Delta_{P Q, R S}^{-1(1)} \gamma_{B, R S}^{(0)}+\gamma_{A, P Q}^{(0)} \Delta_{P Q, R S}^{-1(2)} \gamma_{B, R S}^{(0)}\right] G_{A B} .
\end{aligned}
$$

Here $\gamma^{(0)}, \gamma^{(1)}$ and $\Delta^{-1(1)}$ is given in (24),(34) and (36) and $\Delta^{-1(2)}$ is given by

$$
\Delta^{-1(2)}=-\Delta^{-1(0)} \Delta^{(2)} \Delta^{-1(0)}+\Delta^{-1(0)} \Delta^{(1)} \Delta^{-1(0)} \Delta^{(1)} \Delta^{-1(0)}
$$

In order to obtain the RHS of (39), we need to evaluate $\frac{\delta^{2} \Gamma^{(3)}}{\delta \phi_{A} \delta \phi_{B}}, \gamma^{(2)}$ and $\Delta^{(2)}$ from $\Gamma^{(3)}[\phi, G]$. We have considered only cubic and quartic vertices for simplicity and the results are given in Table 1. By substituting the results of Table 1 to RHS of (39) we obtain

$$
\begin{aligned}
& \Gamma^{(4)}[\phi, G]=\frac{1}{8} \bigoplus_{-\frac{1}{16}} \square-\frac{1}{8} \square_{-\frac{1}{12}}^{\square} \\
& +\frac{1}{48} \square+\frac{1}{48} \longrightarrow-\frac{1}{8} \longrightarrow-\frac{1}{72}
\end{aligned}
$$

which agrees with the previous result given in [10].

\section{DISCUSSIONS AND CONCLUSIONS}

In this paper, we have obtained the recursion relation to obtain the Feynman diagrams of the CJT effective action by using the functional derivative identities. We have shown the two-particle-irreducibility of the CJT effective action and have obtained a Feynman diagrams of the CJT effective action up to the four-loop order. The result agrees with the results obtained previously by extracting 2PI Feynman diagrams from the 1PI effective action. The extension of the method we have used in this paper to the case of recursive generation of the Feynman diagrams of the effective action obtained from the higher order Legendre transformation of the generating functional is in progress. 


\section{Acknowledgments}

This research was supported in part by the Institute of Natural Science.

[1] For a review and references, see M. Sher, Phys. Rep. 179, 273 (1989).

[2] J.M.Cornwall, R.Jackiw and E.Toumboulis, Phys. Rev. D10,2428 (1974).

[3] M. Bachmann, H. Kleinert, A. Pelster, Phys.Rev. D61, 085017 (2000).

[4] B. Kastening, Phys. Rev. E 61, 3501 (2000).

[5] H. Kleinert, A. Pelster, B. Kastening, M. Bachmann , Phys.Rev. E62, 1537 (2000).

[6] A. Pelster, H. Kleinert, M. Bachmann , Annals Phys. 297, 363 (2002).

[7] H. Kleinert, A. Pelster, B. Van den Bossche ,Physica A312, 141 (2002).

[8] A. Pelster, H. Kleinert, Physica A323, 370 (2003)

[9] A. Pelster, K. Glaum, Physica A335, 455 (2004).

[10] K. Kajantie, M. Laine and Y. Schroder, Phys.Rev. D65 045008 (2002).

[11] C. Kim, Phys.Rev. D72 085007 (2005).

[12] C. Kim, Phys.Rev. D74 067702 (2006).

[13] K. Farakos, K.Kajantie, Rummukainen and Y.Schroder, Nucl. Phys. B442, 317 (1995).

[14] S. Coleman and E. Weinberg, Phys. Rev. D7, 1888 (1973).

\section{TABLES}

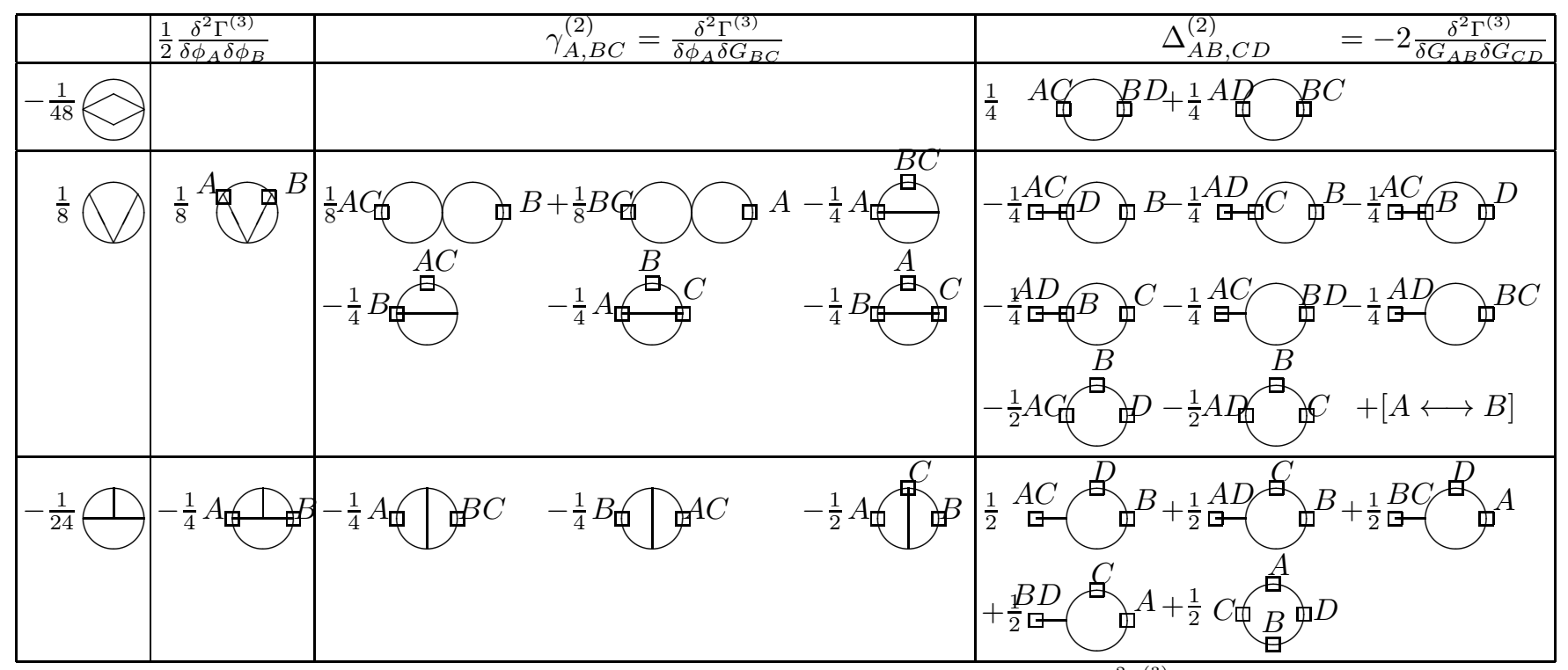

TABLE 1. Contributions from each diagram of $\Gamma^{(3)}[\phi, G]$ ( first column ) to $\frac{1}{2} \frac{\delta^{2} \Gamma^{(3)}}{\delta \phi_{A} \delta \phi_{B}}, \gamma^{(2)}$ and $\Delta^{(2)}$. 


\section{FIGURE CAPTIONS}

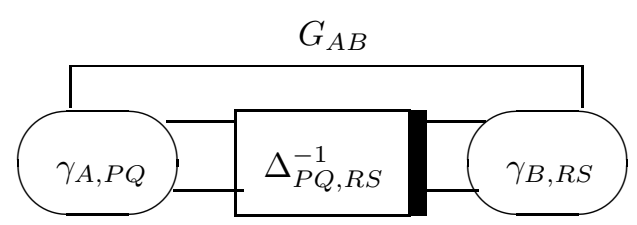

Fig.1. Graphical representation of second term of RHS of (26). Here, a line represents the propagator $G_{A B}$.

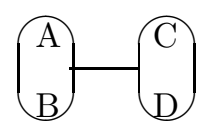

Fig.2. Type of a diagram which is not contained in $\Delta_{A B, C D}$ and $\Delta_{A B, C D}^{-1}$. 